\title{
PLANAR LINE FAMILIES. II
}

\section{PRESTON C. HAMMER AND ANDREW SOBCZYK}

1. Introduction. An outwardly simple planar line family has been defined in the first paper of this series [1] to be a family of lines which simply covers the exterior of some circle, including points at infinity. In [1] a characterization of outwardly simple line families is given and a construction method presented for all constant diameterlength convex bodies in the plane.

In this paper we give a characterization of outwardly simple line families when represented in essentially normal form as a oneparameter family of lines. The internal complexity of an outwardly simple line family is then studied. Two classes of points, the contact points and the turning points of a line family, are defined. The union of these classes forms a generalized envelope of the line family. It is shown, in particular, that the set of all points lying either on an even number of lines or on an infinite number of lines in an outwardly simple line family is of planar measure zero. The set of all intersection points of pairs of lines in an outwardly simple line family is shown to be convex relative to the line family.

2. Characterization of outwardly simple line families. Let $F$ be an outwardly simple line family and let $\beta$ be a circle of radius $r$ containing in its interior all intersection points of pairs of lines in $F$. Let a cartesian coordinate system, $(u, v)$, be chosen so that the origin is at the center of $\beta$. It is shown in [1] that $F$ may be represented by the one-parameter family:

$$
u \sin \alpha-v \cos \alpha-p(\alpha)=0, \quad 0 \leqq \alpha \leqq \pi,
$$

where $p(\alpha)$ is necessarily continuous and $p(0)=-p(\pi)$. These restrictions do not suffice to insure that all intersection points of pairs of lines in $F$ are interior to $\beta$. For purposes of discussion, it is convenient to extend the domain of $p(\alpha)$ by the definition

$$
p(\alpha+\pi)=-p(\alpha), \quad-\infty<\alpha<\infty .
$$

Then lines $m(\alpha)$ and $m(\alpha+\pi)$ in $F$ coincide. Let $\alpha_{1}$ and $\alpha_{2}$ be two distinct angles, $0 \leqq \alpha_{2}-\alpha_{1}<\pi$, and let $u\left(\alpha_{1}, \alpha_{2}\right)$ and $v\left(\alpha_{1}, \alpha_{2}\right)$ be the coordinates of the intersection points of distinct lines $m\left(\alpha_{1}\right)$ and $m\left(\alpha_{2}\right)$. Then, from (1) we have

Presented to the Society, September 4, 1951; received by the editors September 28, 1951 and, in revised form, July 20, 1952. 


$$
\begin{aligned}
u\left(\alpha_{1}, \alpha_{2}\right) & =\left[p\left(\alpha_{2}\right) \cos \alpha_{1}-p\left(\alpha_{1}\right) \cos \alpha_{2}\right] / \sin \left(\alpha_{2}-\alpha_{1}\right), \\
v\left(\alpha_{1}, \alpha_{2}\right) & =\left[p\left(\alpha_{2}\right) \sin \alpha_{1}-p\left(\alpha_{1}\right) \sin \alpha_{2}\right] / \sin \left(\alpha_{2}-\alpha_{1}\right) .
\end{aligned}
$$

Now since all points $\left(u\left(\alpha_{1}, \alpha_{2}\right), v\left(\alpha_{1}, \alpha_{2}\right)\right)$ are interior to $\beta$, we have

$$
\begin{aligned}
r^{2}>u^{2}\left(\alpha_{1}, \alpha_{2}\right)+\nabla^{2}\left(\alpha_{1}, \alpha_{2}\right)= & \csc ^{2}\left(\alpha_{2}-\alpha_{1}\right)\left[p^{2}\left(\alpha_{2}\right)+p^{2}\left(\alpha_{1}\right)\right. \\
& \left.-2 p\left(\alpha_{2}\right) p\left(\alpha_{1}\right) \cos \left(\alpha_{2}-\alpha_{1}\right)\right] .
\end{aligned}
$$

This inequality may be written

$$
r^{2}>\left[\frac{p\left(\alpha_{2}\right)-p\left(\alpha_{1}\right)}{\sin \left(\alpha_{2}-\alpha_{1}\right)}\right]^{2}+2 p\left(\alpha_{2}\right) p\left(\alpha_{1}\right) \frac{\left[1-\cos \left(\alpha_{2}-\alpha_{1}\right)\right]}{\sin ^{2}\left(\alpha_{2}-\alpha_{1}\right)} .
$$

Now, for $0<\alpha_{2}-\alpha_{1} \leqq \pi / 2$ the numerical value of the second term on the right in (5) is bounded and hence the first must also be, i.e.,

$$
\left|p\left(\alpha_{2}\right)-p\left(\alpha_{1}\right)\right|<k_{1}\left|\sin \left(\alpha_{2}-\alpha_{1}\right)\right|<k_{1}\left|\alpha_{2}-\alpha_{1}\right| \text {. }
$$

However, since $p(\alpha)$ is bounded we have that the Lipschitz condition

$$
\left|p\left(\alpha_{2}\right)-p\left(\alpha_{1}\right)\right|<k_{2}\left|\alpha_{2}-\alpha_{1}\right|
$$

necessarily holds for $0<\alpha_{2}-\alpha_{1} \leqq \pi$. A rearrangement of (4) gives

$$
\left|p\left(\alpha_{2}\right)+p\left(\alpha_{1}\right)\right|<k_{3}\left|\pi-\left(\alpha_{2}-\alpha_{1}\right)\right| \text {. }
$$

This inequality is a necessary part of the Lipschitz condition in this instance.

TheOREM 1. Necessary and sufficient conditions that the line family $F$ given by

$$
u \sin \alpha-v \cos \alpha-p(\alpha)=0, \quad 0 \leqq \alpha \leqq \pi,
$$

be an outwardly simple family are that $p(\alpha)$ be continuous, $p(0)$ $=-p(\pi)$, and that $p(\alpha)$ satisfy the Lipschitz conditions (7) and (8).

Proof. The necessity of the conditions has been demonstrated. We write the Lipschitz condition (6) as

$$
p\left(\alpha_{2}\right)=p\left(\alpha_{1}\right)+g\left(\alpha_{1}, \alpha_{2}\right)\left(\alpha_{2}-\alpha_{1}\right) \quad \text { where }\left|g\left(\alpha_{1}, \alpha_{2}\right)\right|<k_{2}
$$

and then substitution in (3) gives

$$
\begin{aligned}
\left|u\left(\alpha_{2}, \alpha_{1}\right)\right| \leqq & 2\left|p\left(\alpha_{1}\right)\right|\left|\frac{\sin \frac{\alpha_{1}+\alpha_{2}}{2} \sin \frac{\alpha_{2}-\alpha_{1}}{2}}{\sin \left(\alpha_{2}-\alpha_{1}\right)}\right| \\
& +\left|g\left(\alpha_{1}, \alpha_{2}\right)\right|\left|\frac{\left(\alpha_{2}-\alpha_{1}\right) \cos \alpha_{1}}{\sin \left(\alpha_{2}-\alpha_{1}\right)}\right|
\end{aligned}
$$


Since the two terms on the right are bounded for $0<\alpha_{2}-\alpha_{1} \leqq \pi / 2$, $\left|u\left(\alpha_{1}, \alpha_{2}\right)\right|$ is bounded in this case.

A similar development using (8) gives that $\left|u\left(\alpha_{1}, \alpha_{2}\right)\right|$ is bounded for $\pi / 2 \leqq \alpha_{2}-\alpha_{1}<\pi$. Likewise $\left|v\left(\alpha_{1}, \alpha_{2}\right)\right|$ is bounded and hence there exists a circle containing all intersection points of $F$ in its interior. Since any family of lines (9) with $p(\alpha)$ continuous in $[0, \pi]$ and $p(0)=-p(\pi)$ covers the plane (including infinite points), $F$ must be outwardly simple. Q.E.D.

3. Turning points and contact points. Let $F$ be a family of lines given by

$$
u \sin \alpha-y \cos \alpha-p(\alpha)=0
$$

where we shall assume initially only that $p(\alpha)$ is continuous for $-\infty<\alpha<\infty$. Then the formulas (3) still hold whenever lines $m\left(\alpha_{1}\right)$ and $m\left(\alpha_{2}\right)$ intersect in a finite point. If $\alpha_{2}=\alpha_{1}+j \pi$ the lines are parallel. Now in (3) let $\alpha_{2}=\alpha+h_{n}, \alpha_{1}=\alpha$, and let $\left\{h_{n}\right\}$ be a null sequence of numbers, either all positive or all negative, over which the difference quotient of $p(\alpha)$ approaches a limiting value (including $+\infty$ and $-\infty$ as possible values). We designate such a limiting value by $D p$. Then from (3) we find for the limit of intersection points on $m(\alpha)$

$$
u(\alpha)=D p \cos \alpha+p \sin \alpha, \quad v(\alpha)=D p \sin \alpha-p \cos \alpha .
$$

If $D p$ is finite, then $(u(\alpha), v(\alpha))$ is a finite point on $m(\alpha)$. Moreover, in that case

$$
u(\alpha) \cos \alpha+v(\alpha) \sin \alpha-D p=0 .
$$

If the derivative $p^{\prime}=D p$ exists for a value of angle $\alpha$, then we obtain (14) by differentiating (12) partially with respect to $\alpha$. If, in addition, $p^{\prime}(\alpha)$ is a continuous function of $\alpha$, then (13) gives the envelope of $F$. We call any finite point $(u, v)$ with coordinates given by (13) a turning point of $F$ on $m(\alpha)$. If $p^{\prime}(\alpha)$ exists, there is a unique turning point of $F$ on $m(\alpha)$ and this point may be considered as an instantaneous center of rotation of $F$.

Consider the continuous function

$$
f(\alpha)=u \sin \alpha-v \cos \alpha-p(\alpha)
$$

for a fixed point $(u, v)$. We shall say that $F$ contacts $(u, v)$ at $\alpha=\alpha_{0}$ or that $(u, v)$ is a contact point of $F$ on $m\left(\alpha_{0}\right)$ if and only if $f\left(\alpha_{0}\right)=0$ and there exists an $\eta>0$ such that either $f\left(\alpha_{0}+h\right)>0, f\left(\alpha_{0}-h\right)>0$ or $f\left(\alpha_{0}+h\right)<0, f\left(\alpha_{0}-h\right)<0$ for $0<h<\eta$. That is, $(u, v)$ is a contact point on $m\left(\alpha_{0}\right)$ if and only if $\alpha_{0}$ is a zero and an isolated relative ex- 
treme point of $f(\alpha)$. In case $p^{\prime}\left(\alpha_{0}\right)$ exists, then the differential condition for a relative extreme of $f(\alpha)$ shows that a contact point is necessarily a turning point. If the derivative of $p$ does not exist at $\alpha_{0}$ and if there is an open interval on $m\left(\alpha_{0}\right)$ between all the left turning points and all the right turning points, every point in the open interval is necessarily a contact point of $F$. (The right turning points are limits of intersection points of $m\left(\alpha_{0}+h\right)$ and $m\left(\alpha_{0}\right)$ as $h \searrow 0$.) For a simple example, the extended diameter family of a triangle has a unique turning point on each line except for the extended sides. Each extended side has the two vertices as turning points. The points between a pair of vertices are all contact points on that extended side.

In case the line family $F$ is outwardly simple, then $p(\alpha)$ satisfies the Lipschitz condition and hence $p^{\prime}(\alpha)$ exists for all angles except those in a set of measure zero. Moreover, $\left|D_{p}\right|$ is uniformly bounded so that the set of turning points of $F$ is bounded.

TheOREM 2. An outwardly simple line family has a unique turning point on almost all of its lines.

4. The measure of the set of turning points. The turning points together with the contact points form a generalized envelope of a line family. One would hope that this set of points is of planar measure zero and it is the object of this section to prove that this is the case for outwardly simple line families and more general line families. For convenience, we initially discuss the outwardly simple line family $F$ given by $u \sin \alpha-v \cos \alpha-p(\alpha)=0,0 \leqq \alpha \leqq \pi$, with turning points $(u, v)$ given by

$$
u=D p \cos \alpha+p \sin \alpha, \quad v=D p \sin \alpha-p \cos \alpha .
$$

Now let the $x$-axis coincide with the $u$-axis and the $y$-axis coincide with the $v$-axis. Define a transformation from the $(x, y)$-plane to the $(u, v)$-plane by

$$
u=x+p \sin \alpha, \quad v=y-p \cos \alpha,
$$

where $\alpha$ is the angle, $\alpha<\pi$, between the $x$-axis and the line through $(0,0)$ and $(x, y)$, and $p=p(\alpha)$. To each point $(x, y)$ except $(0,0)$ there corresponds precisely one point $(u, v)$. This transformation amounts to translating each line in the pencil through $(0,0)$ to the parallel line $m(\alpha)$ in $F$, so that the origin is translated to the point on $m(\alpha)$ nearest $(0,0)$. Now let $S$ be the set of turning points of $F$ and let $S_{0}$ be the set of all points in the $(x, y)$-plane which map into $S$. These points of $S_{0}$ are given by $x=D p \cos \alpha, y=D p \sin \alpha$. Since $D p(\alpha)$ is measur- 
able, it follows that the set of points $S_{0}$ is of planar measure zerousing the polar coordinate area formula $(1 / 2) \int(D p)^{2} d \alpha$. Now we shall indicate the proof of

LEMMA 1. The transformation from the $(x, y)$-plane to the $(u, v)$-plane maps sets of measure zero into sets of measure zero.

Proof. If we let $\left(x_{0}, y_{0}\right)$ be a point at a distance $r_{0}>0$ from $(0,0)$ and take a circle with radius $r<r_{0}$ about $\left(x_{0}, y_{0}\right)$, then this circle maps into the interior of a circle with center $\left(u_{0}, v_{0}\right)$ and radius

$$
R=\left(r+k \arcsin \frac{r}{r_{0}}\right)<r\left(1+\frac{2 k}{r_{0}}\right)
$$

where

$$
\begin{array}{r}
k \geqq\left|\frac{p\left(\alpha_{2}\right)-p\left(\alpha_{1}\right)}{\alpha_{2}-\alpha_{1}}\right| \text { (Lipschitz condition), and } u_{0}=x_{0}+p\left(\alpha_{0}\right) \sin \alpha_{0}, \\
v_{0}=y_{0}-p\left(\alpha_{0}\right) \cos \alpha_{0} .
\end{array}
$$

Hence every open circle of radius $r$ exterior to the circle $C_{0}$ of radius $r_{0}$ and center $(0,0)$ maps into a subset of a circle with a radius no greater than $r\left(1+2 k / r_{0}\right)$. Therefore every set of measure zero exterior to $C_{0}$ is transformed into a set of measure zero. However, this implies that every set of measure zero not including the origin is mapped into a set of measure zero. The origin, however, is mapped into a continuous curve $u=p \sin \alpha, v=-p \cos \alpha$ and this set of points also has measure zero. Hence the lemma is proved.

TheOREM 3. The measure of the set of turning points of an outwardly simple line family $F$ is zero. The measure of the set of contact points of $F$ is zero.

Proof. Lemma 1 shows that the set of turning points is of measure zero. The set of contact points which are not turning points is contained in the subfamily of lines for which $p^{\prime}(\alpha)$ does not exist and these are of measure 0 in $\alpha$. Hence the set of contact points is of measure zero in the plane.

5. Remarks. The proof that the turning points and contact points constitute a set of measure zero does not depend on the outward simplicity of $F$. We shall state a more general result for reference.

THEOREM 4. Let $F$ be a family of lines represented by

$$
u \sin \alpha-v \cos \alpha-p(\alpha)=0
$$


where $-\infty<\alpha<\infty$ and $p(\alpha)$ satisfies a Lipschitz condition in every finite interval. Then the set of turning points and contact points of $F$ is of planar measure zero.

6. Decomposition of the plane. Let $F$ be an outwardly simple line family and let $S_{i}$ be the set of points lying on precisely $i$ lines in $F$ where $i=0,1, \cdots, \infty$. We have shown in [1] that $S_{0}$ is empty.

THEOREM 5. The set of points $S_{2 k}$ and the set of points $S_{\infty}$ are each of planar measure zero. Hence the set of all points each of which is precisely evenly or infinitely covered by $F$ is of measure zero.

Proof. We first show that every point $(u, v)$ in $S_{2 k}$ is a contact point of $F$. Consider

$$
f(\alpha)=u \sin \alpha-v \cos \alpha-p(\alpha) .
$$

Since $(u, v)$ is on precisely $2 k$ lines of $F$, we have that there exist $2 k$ angles $\alpha_{j}$ such that $\alpha_{j}>\alpha_{j-1}, j=1, \cdots, 2 k$, and $f\left(\alpha_{j}\right)=0$. We may assume $f(0) \neq 0$. Now $f(0)=-f(\pi)$, and the angles $\alpha_{j}$ divide the interval $[0, \pi]$ into $2 k+1$ intervals on each of which the function $f$ has one sign. However, $f$ must then have the same sign on two "adjacent" open intervals or $f(0)$ could not be $-f(\pi)$. Hence there exists a value of $\alpha_{j}=\alpha_{0}$ such that $f\left(\alpha_{0}\right)=0$ and the values of $f$ in the two neighboring intervals have the same sign. Then $(u, v)$ is a contact point of $F$ on $m\left(\alpha_{0}\right)$. Since the set of all contact points of $F$ is of measure zero, the set $S_{2 k}$ is then a set of measure zero.

Let $(u, v)$ be a point in $S_{\infty}$. Then the lines in $F$ through $(u, v)$ have a subset converging (in the angle topology) to a line $m\left(\alpha_{0}\right)$. The point $(u, v)$ is necessarily on $m\left(\alpha_{0}\right)$ and hence it is a turning point on $m\left(\alpha_{0}\right)$. But the set of all turning points is of measure zero. Hence the measure of $S_{\infty}$ is zero. Q.E.D.

The class of all turning points and contact points of $F$ is generally not closed. However, we may state

THEOREM 6. On each line $m\left(\alpha_{0}\right)$ in an outwardly simple line family $F$ the set of turning points and contact points at $\alpha_{0}$ is either a closed interval or a single point. If two points on $m$ are intersection points of lines in $F$, then every point on the segment between them is an intersection point. Hence every point simply covered by $F$ is the origin of a half-line on $a$ line of $F$ which is intersected by no other line in $F$.

Proof. Let $D^{+} p$ and $D^{-} p$ represent right and left limits of differential coefficients of $p$ at $\alpha_{0}$. Then the range of $D^{+} p$ and of $D^{-} p$ may be either a single number or a closed interval, since $p$ satisfies the Lipschitz condition. The totality of turning points $(u, v)$ on $m\left(\alpha_{0}\right)$ 
are given by the solutions $(u, v)$ of the equations

$u \sin \alpha_{0}-v \cos \alpha_{0}-p\left(\alpha_{0}\right)=0, \quad u \cos \alpha_{0}+v \sin \alpha_{0}-D p\left(\alpha_{0}\right)=0$.

Hence the set of turning points on $m\left(\alpha_{0}\right)$ is the union of two closed line segments, including single points as degenerate line segments. Suppose that $(u, v)$ on $m\left(\alpha_{0}\right)$ is a contact point of $F$. Then if $f\left(\alpha_{0}+h\right)$ $>0, f\left(\alpha_{0}-h\right)>0$ we have that

$$
D^{-} p\left(\alpha_{0}\right) \leqq u \cos \alpha_{0}+v \sin \alpha_{0} \leqq D^{+} p\left(\alpha_{0}\right) .
$$

If $f\left(\alpha_{0}+h\right)<0$ and $f\left(\alpha_{0}-h\right)<0$ then the inequality signs are reversed. That is, if $m\left(\alpha_{0}\right)$ has a contact point of $F$ on it, it must coincide with a unique turning point or it lies "between" all the right turning points and all the left turning points. Conversely, all points on an open line segment separating the right turning points and left turning points on $m\left(\alpha_{0}\right)$ are contact points. The union of the sets of turning points and contact points of $F$ on $m\left(\alpha_{0}\right)$ is thus either a closed line segment or a single point.

For the remainder of the proof, it will suffice to show that any point on a line in $F$ between two intersection points of lines in $F$ is necessarily covered at least twice by $F$. This is implied by the following lemma.

LEMMA 2. Let $m_{1}, m_{2}$, and $m_{3}$ be any three lines in $F$ which are not copunctal. Then the points interior to the triangle formed by $m_{1}, m_{2}, m_{3}$ are covered at least thrice by $F$ and the sides of the triangle are covered at least twice.

Proof. Let $m_{1}$ and $m_{2}$ be any two lines in $F$. We may suppose that $m_{1}$ corresponds to $\alpha=0$ and $m_{2}$ to $\alpha=\alpha_{0}>0$. We may also assume the origin to be at the intersection of $m_{1}$ and $m_{2}$. We shall show that the lines $m(\alpha)$ in $F, 0<\alpha<\alpha_{0}$, cover every point in the two infinite sectors from $m_{1}$ to $m_{2}$. To do this, consider the line family $F$ as given by zeroes of

$$
f(\alpha)=u \sin \alpha-v \cos \alpha-p(\alpha) .
$$

Let $(\rho, \theta)$ be the polar coordinates of a point in the $(u, v)$-plane such that $\rho>0,0<\theta<\alpha_{0}$. Then the cartesian coordinates of this point are $\rho \cos \theta, \rho \sin \theta$. Using $f(\alpha)$ for these values for $u$ and $v$, we have

$$
f(\alpha)=\sin (\rho-\theta)-p(\alpha) .
$$

Now $p(0)=p\left(\alpha_{0}\right)=0$ and hence

$$
f(0)=-\rho \sin \theta, \quad f\left(\alpha_{\theta}\right)=\rho \sin \left(\alpha_{0}-\theta\right) .
$$


Hence, $f(0)<0$ and $f\left(\alpha_{0}\right)>0$ and there exists a value of $\alpha=\alpha_{1}$ such that $0<\alpha_{1}<\alpha_{0}$ and $f\left(\alpha_{1}\right)=0$. The point $(\rho, \theta)$ is on $m\left(\alpha_{1}\right)$. Similarly, every point of form $(\rho, \theta)$ where $\rho<0,0<\theta<\alpha_{0}$, is on a line $m\left(\alpha_{2}\right)$ in $F$ such that $0<\alpha_{2}<\alpha_{0}$. Thus the triangle with extended sides $m_{1}, m_{2}$, and $m_{3}$ is covered minimally as described.

To complete the proof of the theorem, if a point lies on a line $m_{1}$ in $F$ between two intersection points on that line, then we have two additional lines in $F, m_{2}$ and $m_{3}$, determining a triangle as required by the lemma. Hence the point is covered at least twice by $F$. Q.E.D.

7. Remarks. Theorem 6 establishes that every line in $F$ is divided into two infinite rays each of which is not intersected by other lines in $F$ and a line segment which may be open, closed, half-closed or a single point which separates the rays and contains all intersections of the line with others in $F$. The points simply covered by $F$ are accessible to the exterior along a ray of a line in $F$. The set of all turning points and contact points of $F$ is convex and closed relative to $F$. This property is akin to starlikeness of regions but is more general in that it refers to outwardly simple line families rather than pencils of lines.

8. Conclusion. In this paper we have given additional necessary and sufficient conditions for a planar line family to be outwardly simple. The sets of turning points and contact points give a generalized envelope of such a line family. This set is shown to be of measure zero.

There are numerous modes of generalization which are suggested by this treatment. We have made an initial study of outwardly simple continuous line families in higher dimensions. In Planar line families. III, a characterization of continuous outwardly $k$-fold coverings of the plane by line families is given. Another form of extension under consideration is the covering of lines in 3-space by planes and generalizations of this form to higher dimensions.

Appendix A. Examples of some of the principles stated in the paper may be useful. For a regular polygon with an odd number $2 n+1$ of sides, the extended diameters form an outwardly simple family of lines. All the turning points are the vertices and all the contact points are those on the open interval on line segments joining each vertex to the end points of the opposite side. Except for intersections with other such open intervals the contact points are evenly covered by the diameter family. The set $S_{2 n+1}$ has a positive area as do all sets $S_{2 k+1}$ for $0 \leqq k<n$ where $n \geqq 2$.

One might expect that an outwardly simple family must cover at 
least one point an infinite number of times. That this is not the case is shown by the following example of an outwardly simple family which covers any point a maximum of three times.

Let $a:(0,-\sin \theta)$ and $b:(\cos \theta, \sin \theta)$ be two points in the cartesian coordinate system. As $\theta$ varies from $+\pi / 2$ to $-\pi / 2, a$ moves from $(0,-1)$ to $(0,1)$ on the $y$ axis and $b$ describes a unit semicircle in the right half-plane. The family of lines obtained by extending the line segments $a b$ is an outwardly simple family given by the one-parameter family $y+\sin \theta=2 x \tan \theta$. In terms of the angle $\alpha$ used in the paper this family can be represented by

$$
x \sin \alpha-y \cos \alpha-\frac{\sin \alpha}{\left(4+\tan ^{2} \alpha\right)^{1 / 2}}=0
$$

where $-\pi / 2 \leqq \alpha \leqq \pi / 2$. For a fixed $x>0$ consider the equation $y$ $=2 x \tan \theta-\sin \theta$. Considered in the $\theta y$-plane the curve is symmetric with respect to the origin and has relative extremes for $\theta= \pm \cos ^{-1}(2 x)^{1 / 3}$ if $0<2 x<1$. If $2 x \geqq 1$ the curve is monotone increasing, and then for every value of $x$ there is only one value of $y$ for each value of $\theta$. If $0<2 x<1$, then for values of $y$ between the relative maximum value and the relative minimum, there are precisely three distinct angles $\theta$ giving that value of $y$. For $y$ equal to a relative minimum or maximum there are two angles $\theta$ giving that value of $y$. For each $y$ above or below the extreme values there is precisely one angle $\theta$ giving that value of $y$. The set of points doubly covered by the family are those on the open interval from $(0,-1)$ to $(0,1)$ and those on the curves

$$
y= \pm\left[1-(2 x)^{2 / 3}\right]^{3 / 2}
$$

for $0<x<1 / 2$. These latter curves form the envelope of the family of lines. If we include points $(1 / 2,0),(0,-1)$, and $(0,1)$ with the doubly covered points we have a simple closed curve containing all triply covered points in its interior. The points exterior to the curve are simply covered. In view of Lemma 2, this example gives the smallest maximal coverage of points obtainable from any outwardly simple line family.

\section{REFERENCES}

1. P. C. Hammer and A. Sobczyk, Planar line families. I, Proceedings of the American Mathematical Society vol. 4 (1953) pp. 226-233.

2. P. C. Hammer, Convex bodies associated with a convex body, Proceedings of the American Mathematical Society vol. 2 (1951) pp. 781-793.

Los Alamos Scientific Laboratory 